\title{
Percepción y actitudes frente al acoso sexual callejero en estudiantes mujeres de una Universidad Privada de Medicina
}

Ruth Corazón Llerena Benites ${ }^{1}$

RESUMEN

Objetivo: Determinar la percepción y las actitudes frente al acoso sexual callejero que tienen las estudiantes mujeres del bloque clínico de la Facultad de Medicina Humana de la Universidad San Martín de Porres.

Material y Métodos: Estudio descriptivo de corte transversal en el cual se aplicaron los cuestionarios, tipo Likert previamente validados, la "Escala de aceptación de mitos modernos sobre la agresión sexual" y la "Escala de acoso callejero", de forma virtual a 227 estudiantes mujeres pertenecientes al 4to, 5to y 6to año académico de la Facultad de Medicina Humana de la Universidad San Martín de Porres. El análisis se realizó en SPSS v22, con estadística descriptiva utilizando medias, tablas de frecuencias y porcentajes para determinar la prevalencia del acoso callejero y el nivel de aceptación de creencias sobre agresión sexual.

Resultados: $91 \%$ de las participantes consideraban haber sido acosadas al menos una vez en el pasado año. $48 \%$ del total de participantes estaba totalmente en desacuerdo con las afirmaciones acerca de los mitos sobre agresión sexual. El porcentaje de participantes que referían nunca haber sido acosadas, disminuía al examinar cada sección de estudios, comenzando con un $13 \%$ del total de 4to año para bajar a $7.9 \%$ en la sección de 6to año. La mayoría provenía de Lima Central Sur, de las cuales el $88 \%$ había sido acosada al menos una vez en el pasado año. Aproximadamente la mitad de las participantes, independiente al medio de transporte que hayan usado, indicaron haber sido acosadas una vez el pasado año. El grupo de 22 años fue el más frecuentemente afectado.

Conclusión: Si bien el promedio de estudiantes refería que el acoso callejero se había limitado de una a algunas veces en el pasado año, no se subestimaba el hecho que para casi el $100 \%$ esto había ocurrido al menos una vez. También la mayoría se consideraba estar entre muy en desacuerdo a neutral respecto a los mitos sobre agresión sexual. Entonces, se evidencia que el acoso callejero, a pesar de actuar como un problema social por sus repercusiones en el bienestar físico y mental de la población femenina joven, aún no ha sido adecuadamente abarcado.

Palabras clave. Acoso callejero, repercusión social, violencia basada en género, mitos

Perception and attitudes towards street sexual harassment among female students of a
private Human Medicine Faculty. ABSTRACT

Objective: Determinate the perception and attitudes towards street sexual harassment among female students of the Human Medicine Faculty at San Martin de Porres University.

Methods: Descriptive and transversal study in which the previously validated "Likert" questionnaires, "Scale of Acceptance of Modern Myths about Sexual Aggression" and "Street Harassment Scale" where applied in a virtual way to 227 female students from the 4th, 5th, 6th academic year of the Human Medicine Faculty at San Martin de Porres University. The analysis was made in the SPSS V22 program using descriptive statistics like media, mode, tables of frequency and percentage to determine the prevalence of street harassment and the level of acceptance of beliefs about sexual harassment.

Results: We found that $91 \%$ of the participants considered that they had been (sexually) harassed at least once in the last year. $48 \%$ of participants were absolutely disagree with the statements about the myths of sexual aggression. The percentage of students that mentioned never have been harassed lowered for every year of study, from $13 \%$ in the $4^{\text {th }}$ year to $7.9 \%$ in the $6^{\text {th }}$ year. Most of the students came from Central South Lima of which $88 \%$ were harassed at least once the past year. Approximately, about half of the participants, independent of the mean of transport they have used, said that they had been harassed once last year. The group of 22 years old was the most affected

${ }^{1}$ Estudiante de la FMH USMP

Conclusion: Even though the participants considered that the Street harassment only happened a few times the past year, we didn't underestimate the fact that for almost everyone this harassment had happened at least once. Also, the majority considered to be strongly disagree regarding the myths about sexual harassment. So, it appears that street harassment, despite acting as a social problem that affects the physical and mental well-being of the Young female community, hasn't been properly managed by the authorities yet.

Keywords: Street Harassment, Repercussions, Gender based violence, Myths

\footnotetext{
${ }^{1}$ Estudiante de la FMH USMP
} 


\section{INTRODUCCIÓN}

Según estadísticas a nivel global, a través del Social Index Progress, los países latinoamericanos han sido los que menos han considerado que la mujer es tratada con dignidad y respeto (1).

En el Perú, encontramos la definición de acoso sexual en la Ley de Prevención y Sanción del Hostigamiento Sexual, ley 27942, como "la conducta física o verbal reiterada de naturaleza sexual no deseada o rechazada, que afecta la dignidad, así como los derechos fundamentales de la persona agraviada, que se da en el contexto de relaciones de autoridad o dependencia pero también con prescindencia de jerarquías" (2). El acoso callejero es una de las formas de violencia de género más practicadas en nuestro país, debido a su brevedad de duración, anonimato y poco definida penalización. Tiene como raíz a una sociedad patriarcal jerarquizada que utiliza muchas veces la violencia como medio de dominación masculina simbólica (3).

El acoso callejero es un conjunto de acciones que abarcan desde comentarios, gestos, silbidos, sonidos de besos hasta tocamientos, masturbación pública, exhibicionismo, seguimientos, etc., con insinuación sexual. Estas prácticas revelan relaciones de poder entre géneros, puesto que los hombres son los que suelen ejercerla mayormente sobre mujeres, en la mayoría de casos desconocidas para ellos (4). Gran parte de la población parece estar habituada a dichas acciones y las aceptan como comunes e inofensivas. Esto ha favorecido su "invisibilización" como problema (5). También hay que destacar que algunas formas de acoso callejero son calificadas por las mujeres de distintas maneras. Por ejemplo, la mayoría de mujeres asumen que un comentario en la calle puede ser un piropo en vez catalogarlo como acoso. Esta variación en cómo el acoso callejero es interpretado dificulta conceptualizarlo como una acción violenta y encontrar el mejor método para responder a este comportamiento (6).

A nivel nacional, el trabajo más especializado acerca del acoso callejero fue el realizado por la Pontificia Universidad Católica del Perú, la cual halló que un tercio de las mujeres había sufrido algún tipo de hostigamiento sexual de tipo verbal y que alarmantemente la mayoría de ellas consideraba que su forma de vestimenta era una de los factores. Entre las repercusiones observaron que casi un $70 \%$ de mujeres caminaban temerosas en la calle a cualquier hora del día (5). En el 2012, se realizó un estudio exploratorio acerca del hostigamiento sexual en universitarios a cargo del Ministerio de la Mujer y Poblaciones vulnerables, participando 189 estudiantes de una universidad pública de Lima. Entre los resultados destacaron que una de tres mujeres había padecido de acoso sexual y que esto había repercutido en su vida diaria y salud mental. Además, que no existía confianza en las instituciones administrativas o judiciales para reportar estos hechos. Concluyeron que era necesario emitir una ley en contra del hostigamiento sexual en nuestro país (2).

A nivel internacional existen diversos estudios que caracterizan con más precisión el acoso callejero, sus consecuencias y la falta de confianza en las autoridades de tomar acciones al respecto. Fairchild y Rudman (2008) estudiaron el acoso callejero y la objetificación de la mujer en 228 estudiantes mujeres estadounidenses, llegando a concluir que el auto-objetificación y el temor a violación era directamente proporcional a la frecuencia de acoso sexual y también que las mujeres con mayor temor a violación restringían su libertad de movimiento (7). Sullivan (2011) del Indiana University of Michigan realizó un trabajo experimental con 79 estudiantes mujeres de su universidad que la mayoría había experimentado frecuentemente de acoso sexual callejero y en mayor grado de tipo menor y que a pesar de que no había diferencias significativas en ambos grupos en cuanto a las perspectivas frente al acoso callejero, las que habían visto el video del acoso presentaban mayor grado de enojo que las que no (8).

Lisa K. Kearney (2004) estudió las diferencias culturales en las respuestas ante el acoso sexual por parte de 111 estudiantes caucásicas y 261 mexicanoamericanas en tres universidades en Texas y México. Determinó que las estudiantes caucásicas padecían de más casos de acoso sexual que las estudiantes mexicanas y que ambos grupos presentaban el mismo perfil de respuesta al acoso lo que hizo concluir que no había diferencias culturales que influyesen la percepción del acoso sexual de esa población (9).

En el 2013, el Instituto de Educación de Hong Kong emitió un reporte sobre las actitudes sexuales y punto de vista del acoso sexual de 5902 estudiantes y profesores de distintos centros educativos, a los cuales se les realizó una encuesta personalizada en focus groups. Encontraron que la mayoría no tomaba en cuenta una política en contra del acoso sexual y si 
Percepción y actitudes frente al acoso sexual callejero en

estudiantes mujeres de una Universidad Privada de Medicina

había, no estaba bien determinada debido en gran parte a que el personal no estaba adecuadamente entrenado y que tampoco consideraban que había urgencia en la realización de dicha política (10).

En el 2014, Herrera et col publicaron un artículo en España acerca de la reacción masculina ante la confrontación del acoso sexual. Participaron en el estudio 101 hombres que contestaron un cuestionario en el que se manipulaban dos de los tipos de acoso más frecuentes así como la respuesta de la víctima. Los resultados resaltaron la dificultad de reconocer determinados comportamientos como acoso, así como las posibles consecuencias que puede sufrir la víctima en función de la respuesta que dé a estas situaciones de acoso. Las estrategias usadas por las mujeres para afrontar el acoso parecían presentar algún obstáculo o problema para ellas, por lo que concluyeron que se hacía necesaria la implantación de programas preventivos y/o educativos con el fin de enseñar a hombres y mujeres a comprender mejor el constructo (11).

Mientras que el acoso callejero es usualmente visto como benigno o poco dañino. Estudios postulan que esas experiencias pueden generar efectos negativos profundos en las víctimas. Por ejemplo, Macmillan at col (2000) encontraron que el acoso callejero impactaba en las percepciones de seguridad de los participantes. Para los participantes en la investigación de Fairchild y Rudman (2008), experimentar acoso sexual por parte de extraños fue relacionado con miedo y percepción de riesgo a violación. Ho at col (2012) relacionaron al acoso sexual con la aparición de síntomas negativos en estudiantes mujeres como ansiedad, miedo, vergüenza, culpa, cefaleas, sueño interrumpido, decremento de apetito y pérdida de peso. También encontraron síntomas relacionados síndrome post traumático. La severidad de éstos, en especial la depresión, estuvieron directamente correlacionados a la frecuencia de la exposición al acoso sexual (12). El acoso sexual callejero tiene el potencial de impactar en la percepción de seguridad en lugares públicos y en limitar su libertad de movimiento y acceso a éste. Dicho impedimento afectaría negativamente su bienestar social y económico (13).

Por todo lo anterior podemos suponer que existe un campo de investigación amplio en lo que se refiere al acoso sexual callejero en una sociedad latinoamericana patriarcal como la nuestra.

\section{MATERIAL Y MÉTODOS}

Es un estudio descriptivo de corte transversal. De una población de 540 mujeres pertenecientes del 4to a 6to año de la facultad de Medicina Humana de la Universidad San Martín de Porres, se obtuvo una muestra de 227 estudiantes. El muestreo fue no probabilístico de tipo casual, por ello carecerá de representatividad.

Para la recolección de datos se promocionó de forma virtual el link de un formulario compuesto por un consentimiento informado y dos cuestionarios. Los instrumentos usados fueron la "Escala de aceptación de mitos modernos sobre la agresión sexual" (14) que consistía en una escala tipo Likert de 30 ítems que evaluaban las creencias populares acerca de la agresión sexual, las estudiantes marcaban de 1 (totalmente en desacuerdo) a 7 (totalmente de acuerdo) con las premisas expuestas; y la "Escala de acoso callejero" (8) , escala de 7 ítems estilo Likert del 0 "Nunca" al 4 "Casi Siempre" que estimaba la frecuencia de acoso sexual callejero. Para la validación de los instrumentos al contexto peruano se realizó consulta con especialistas, revisión de traducción, retrotraducción, focus group virtual con 15 estudiantes del 6 to año de Medicina Humana y se obtuvo un coeficiente alfa de cronbach de 0,71 para la "Escala de acoso callejero" y un 0.86 para la "Escala de aceptación de mitos modernos sobre la agresión sexual.

El análisis se realizó en SPSS v22, con estadística descriptiva utilizando media, desviación estándar, moda, tablas de frecuencias y porcentajes para determinar la prevalencia del acoso callejero y el nivel de aceptación de creencias sobre agresión sexual.

\section{RESULTADOS}

De la población total de estudiantes mujeres del bloque clínico de Medicina Humana $(n=540)$, participaron 227 de las cuales $80.7 \%$ del 6to año, $43.8 \%$ del 5 to año y $7.5 \%$ del 4 to año. Se abarcaron edades desde los 18 hasta los 28 años. Siendo la mayoría (39\%) pertenecientes al grupo del bloque de los 21-25 años. Con respecto a la zona de vivienda, $14.6 \%$ provenían de Lima Central Sur, $11.8 \%$ de Lima Este y el $8.9 \%$ de Lima central. La mayoría (31.5\%) se transportaba en buses o microbuses y en segundo lugar a través de carros propios (9\%). 
Figura 1. Resultado promedio de la escala de acoso callejero

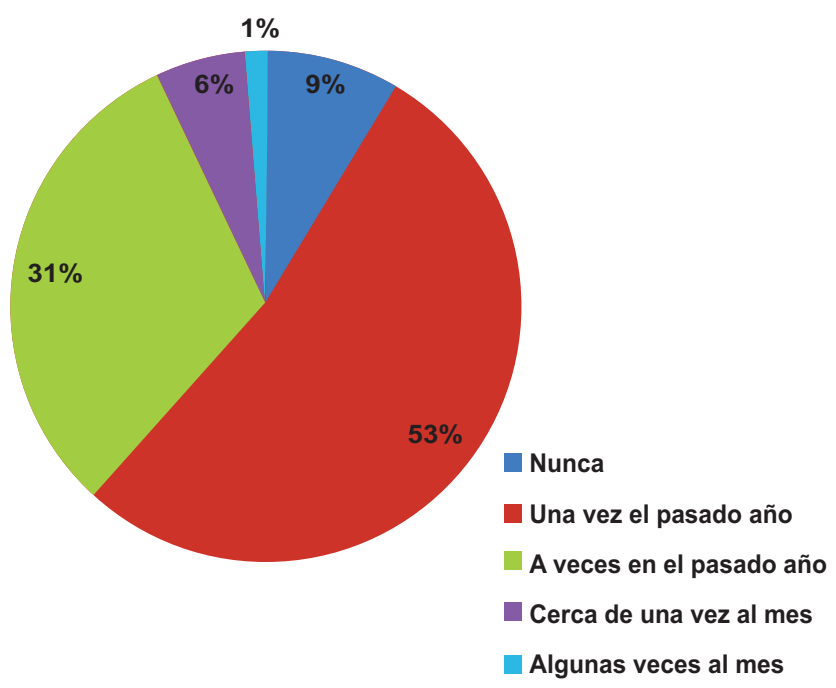

En la figura 1, se ilustra cómo el $91 \%$ de las participantes consideraban haber sido acosadas al menos una vez en el pasado año. Siendo la mayoría (53\%) haber considerado molestadas una vez el pasado año, $31 \%$ algunas veces el pasado año y un $6 \%$ cerca de una vez al mes. Solo $9 \%$ consideraban nunca haber sido acosadas en el medio público.

Figura 2. Resultados de la escala de acoso callejero según sección

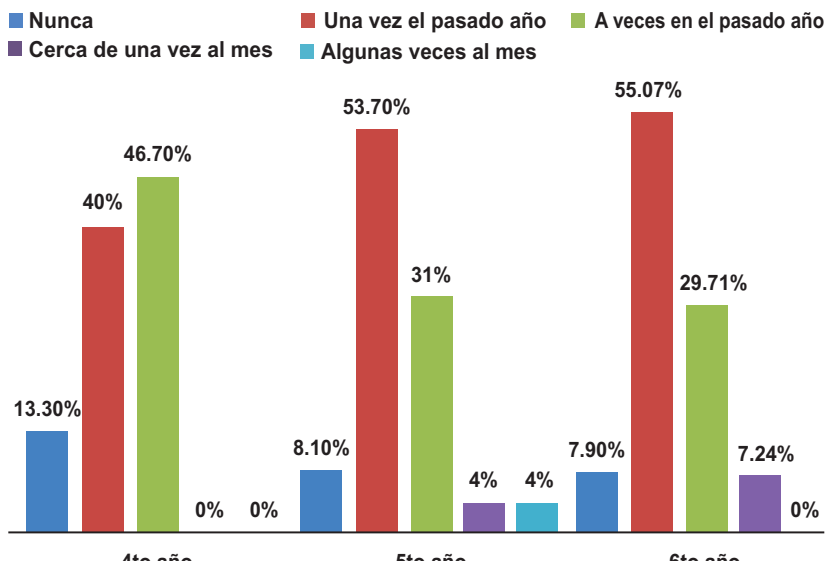

En la figura 2, encontramos que el porcentaje de participantes que señalaban nunca haber sido acosadas disminuía al examinar cada sección de estudios, comenzando con un $13 \%$ del total de 4 to año para bajar a $7.9 \%$ en la sección de 6to año. Lo opuesto ocurre con las participantes que indicaban ser acosadas al menos una vez al mes en el pasado año: el porcentaje de 4 to año comienza con $0 \%$ de su total, mientras que en la sección de 5 to año sube a $4 \%$ y la de 6to año a $7.24 \%$.
Figura 3. Resultado promedio de la escala de acoso callejero según zona de vivienda

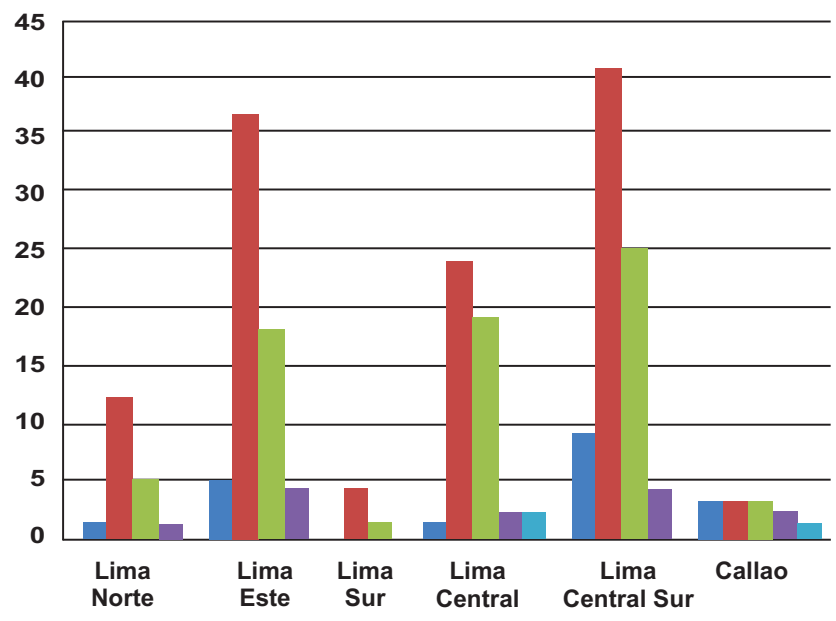

En la figura 3 observamos que la mayor parte de las participantes provenían de Lima Central Sur $(n=79)$, de las cuales el $88 \%(n=70)$ había sido acosada al menos una vez en el pasado año. En Lima Este $(n=64)$ el resultado es similar, el $92 \%(n=59)$ de las participantes de esta zona señalaban haber sido acosada al menos una vez en el pasado año.

Figura 4. Resultado promedio de la escala de acoso callejero según medio de transporte más utilizado.

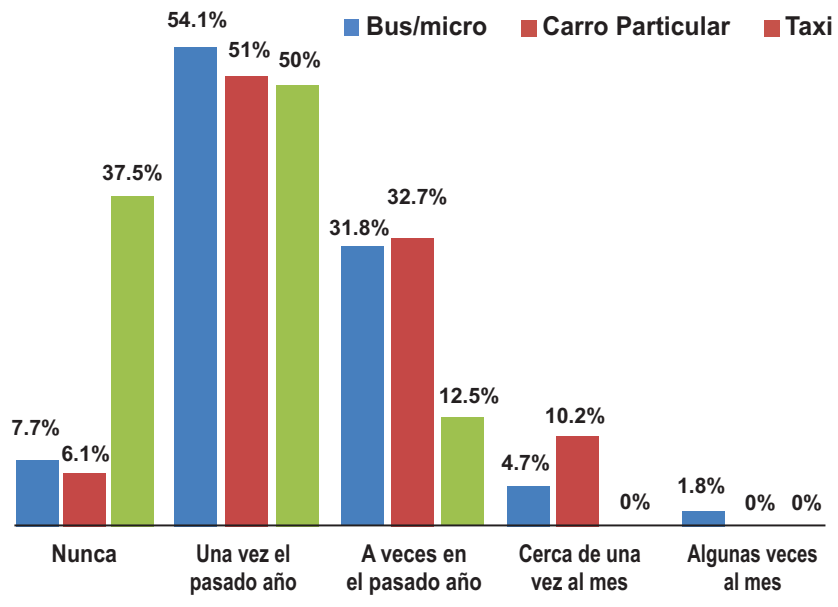

En la figura 4, podemos extraer que aproximadamente la mitad de las participantes, independiente al medio de transporte que hayan usado, indicaron haber sido acosadas una vez el pasado año. También se encontró que más de un tercio $(37.5 \%)$ de las participantes que habían utilizado taxis como medio de transporte frecuente consideraban nunca haber sido víctimas de acoso callejero. 
Tabla 3. Resultado promedio de escala de acoso callejero según edad

\begin{tabular}{|c|c|c|c|c|c|c|}
\hline \multirow{11}{*}{ Edad } & Nunca & $\begin{array}{l}\text { Una vez el } \\
\text { pasado año }\end{array}$ & $\begin{array}{l}\text { A veces en el } \\
\text { pasado año }\end{array}$ & $\begin{array}{l}\text { Cerca de } \\
\text { una vez } \\
\text { al mes }\end{array}$ & $\begin{array}{l}\text { Algunas } \\
\text { veces } \\
\text { al mes }\end{array}$ & Total \\
\hline & 18 & 0 & 0 & 0 & 0 & 1 \\
\hline & 20 & 2 & 5 & 0 & 0 & 8 \\
\hline & 21 & 6 & 19 & 4 & 0 & 40 \\
\hline & 22 & 4 & 40 & 4 & 0 & 68 \\
\hline & 23 & 3 & 26 & 3 & 3 & 54 \\
\hline & 24 & 3 & 23 & 2 & 0 & 36 \\
\hline & 25 & 1 & 5 & 0 & 0 & 12 \\
\hline & 26 & 0 & 1 & 0 & 0 & 3 \\
\hline & 27 & 0 & 2 & 0 & 0 & 2 \\
\hline & 28 & 0 & 0 & 0 & 0 & 2 \\
\hline \multicolumn{2}{|c|}{ Total } & 19 & 121 & 13 & 3 & 226 \\
\hline
\end{tabular}

De la tabla 3, obtenemos que las participantes de 22 años fueron el grupo dominante $(30 \%, n=68)$, así como las que mayormente $(28,2 \%$ del total general) indicaron haber padecido de acoso callejero al menos una vez el pasado año.

Tabla 4. Respuesta mayormente marcada en el AMMSA

\begin{tabular}{|ccc|}
\hline Totalmente en desacuerdo & 110 & 48,5 \\
\hline Muy en desacuerdo & 33 & 14,5 \\
\hline Parcialmente en desacuerdo & 21 & 9,7 \\
\hline Neutral & 36 & 15,9 \\
\hline Parcialmente de acuerdo & 18 & 7,9 \\
\hline Muy de acuerdo & 4 & 1,8 \\
\hline
\end{tabular}

En la tabla 4, observamos que casi la mitad (48\%) de las participantes estaba totalmente en desacuerdo con las afirmaciones acerca de los mitos sobre agresión sexual, mientras que un $16 \%$ se mantuvieron neutrales respecto al tema y un $12 \%$ se inclinaba a estar de acuerdo con las afirmaciones.

Tabla 5. Respuestas mayormente marcadas en cada encuesta

\begin{tabular}{|ccc|}
\hline Total & $\begin{array}{c}\text { Resultado promedio de } \\
\text { escala de acoso callejero }\end{array}$ & $\begin{array}{c}\text { Resultado Promedio de } \\
\text { AMMSA }\end{array}$ \\
\hline Media & 227 & 227 \\
\hline Moda & 1,35 & 2,87 \\
\hline Dev. Standar & $1^{\text {a }}$ & 3 \\
\hline
\end{tabular}

a. Multiple modes exist. The smallest value is shown

En la tabla 5, podemos observar las respuestas mayormente marcadas en cada encuesta. En la escala de acoso callejero, el promedio había considerado haber sido acosada de una a algunas veces el pasado año. Mientras que en el AMMSA, el promedio estaba de muy en desacuerdo a una actitud neutral con respecto a los mitos sobre agresión sexual.

\section{DISCUSIÓN}

En el presente estudio se buscó describir una situación que muchas mujeres jóvenes en el Perú viven continuamente y que a pesar de ser un problema social, que pude afectar la percepción de seguridad y limitar la libertad de movimiento sólo por el género (5), no ha sido más profundamente investigado.

Para esto se eligió a una población femenina con características particulares: estudiante de Medicina Humana de pregrado, que por su misma condición, transita por diferentes zonas de Lima Metropolitana y Callao para acudir a los hospitales de ESSALUD, MINSA, Fuerzas Armadas y Clínicas a los que acuden personas de todos los estratos socioeconómicos. Por ello, se presumió que esta población podría proporcionar una imagen ampliada del "fenómeno" que es el acoso callejero.

Se encontró que, el $91 \%$ de las participantes consideraron haber sido acosadas al menos una vez en el 2014 y que el $84 \%$ refirió que esta situación había ocurrido de una a algunas veces el pasado año. Esto concuerda con el estudio realizado por Sullivan (2011) acerca del acoso callejero en estudiantes mujeres de la Universidad de Indiana en Pensilvania donde halló que el $97 \%$ señalaba haber sido víctima de acoso al menos una vez y el $85 \%$, de una a algunas veces al año(8). Un factor importante a considerar ante estos resultados es que si bien los resultados fueron muy parecidos y se utilizaron los mismos criterios (comentarios sobre imagen personal, sonidos y gestos inapropiados, seguimientos, etc.) para calificar "acoso callejero", aquellos ocurrieron en realidades completamente distintas (país primermundista vs país tercermundista). Si contrastamos con estudios nacionales, en el 2012 el Instituto de Opinión Pública de la Pontificia Universidad Católica del Perú describió que un 68\% de las participantes se sentía insegura en calle a cualquier hora del día y que a un $53 \%$ les habían silbado, observado, seguido, hablado o tocado inapropiadamente (5) . Por tanto, es importante destacar la manera en que se define "acoso callejero" según el contexto cultural. Por ejemplo, Kearney (9) propuso que el concepto de "acoso sexual" podría variar en culturas hispánicas, debido a normas sociales propias de esta etnia, causando una disminución en denuncias de dicho acoso con respecto a la definición de otros países como Estados Unidos. Sin embargo, al comparar los criterios utilizados en los estudios mencionados y sus resultados, podemos concordar con Donovan y Drasgow (15) que, si bien hay diferencias en ciertos comportamientos específicos que son considerados 
acoso según el contexto cultural, situaciones más generales como silbidos, gestos o comentarios inapropiados son criterios comunes para considerar "acoso".

Si analizamos la figura de acoso callejero según año de estudios, hallamos que el porcentaje de participantes que habían señalado nunca haber sido molestadas el pasado año, disminuía a medida que subíamos de sección y lo contrario ocurría cuando observábamos los porcentajes de estudiantes molestadas al menos una vez al mes según sección. Podríamos tomar en cuenta la frecuencia y variedad de movilización por Lima para entender este fenómeno. La sección de 4to año de Medicina se restringía a rotar a un solo hospital todo el año académico, mientras que las secciones de 5to y 6 to año rotaban en varios hospitales, distribuidos por todo Lima y Callao a lo largo el año.

Se describe también que la zona de Lima Metropolitana de la que proviene el porcentaje de estudiantes con mayor frecuencia de incidentes $(30 \%$ del total) fue Lima Central Sur que comprende San Borja, Chorrillos, Surco, Surquillo, Santiago de Surco, San Borja, San Isidro y Miraflores. Casi 35\% de estudiantes habitaban en esta zona y el $88 \%$ de éstas habían sido acosadas al menos una vez. El que le seguía era Lima Este ( $25 \%$ del total), que incluye a los distritos de La Molina, San Juan de Lurigancho, Chaclacayo, Santa Anita, Ate y San Luis, donde provenían casi la tercera parte de las estudiantes de las cuales el $92 \%$ habían sido acosadas al menos una vez en el 2014.

Se postulaba en un principio que habría un alto porcentaje de alumnas acosadas que soliesen utilizar a los buses o microbuses como medio de transporte habitual. Esto se basaba en la impresión de que las circunstancias de anonimato, excesiva o poca cantidad de usuarios e imprecisión del contacto físico dentro de un bus/microbús o en sus paraderos facilitaban la práctica del acoso callejero. No obstante, los resultados manifestaron que sin importar que fuesen usuarias habituales de bus, taxi o carro privado, aproximadamente la mitad de cada grupo habría sido acosada al menos una vez en el pasado año. Mientras que más de un tercio de las participantes que solían usar taxi como medio de transporte habitual, había referido nunca haber sido acosada en el 2014.

Según el estudio realizado por Vallejo Rivera para el movimiento "Paremos el acoso callejero" en Lima, se destacó que las mujeres de entre 18 a 29 años era el segmento más afectado (4), lo que se asimila a nuestra tabla de acoso según edad, hallando que el grupo de 21 a 25 años correspondía al 85\% del total de estudiantes acosadas. Como lo explica la autora , este grupo etario se encuentra adaptándose a su independencia como adulto joven y por tanto a la falta de supervisión adulta, lo que es aprovechado por la mayoría de acosadores que utilizan prácticas socialmente consideradas como "benignas" y sin repercusiones legales. Aunque también propone que "a mayor juventud, mayor posibilidad de ser afectada" (4), en este estudio, abarcando edades de entre 18 a 28 años, el grupo más perjudicado fue el de 22 años (28\% del total de participantes).

El segundo eje de este estudio fue determinar las actitudes con respecto al acoso callejero. Para ello utilizamos la escala de aceptación de mitos modernos sobre agresión sexual $(14,16)$ que nos ofrecía una serie de ejemplos de "creencias descriptivas 0 prescriptivas sobre la violación” $(17,18)$ las cuales sugieren un grado de aceptación a conductas e ideas que violentan la libertad sexual de la mujer. Tal como propone Romero-Sánchez et al (16), estas creencias implican "culpabilizar a la víctima, minimizar el impacto psicológico de la agresión y justificar al agresor", lo que repercutiría en la manera en que las mujeres reconocen una agresión e intenten denunciarla. Nuestros resultados mostraron que el $72 \%$ de estudiantes en distintos grados no estaban de acuerdo con las premisas de la escala, especificando que casi la mitad de la población estaba totalmente en desacuerdo. Aquello también implica que aproximadamente la tercera parte mantuvo una actitud neutral o se inclinaba a estar de acuerdo con las afirmaciones hechas. Para interpretar esta tendencia, podríamos tomar en cuenta que los mitos sobre la violación tienen base en el patriarcalismo típico de la sociedades latinoamericanas, en las cuales es parte del contexto cultural normal el que, el hombre para definir su virilidad, asuma un "poder natural" sobre la mujer (19). Por tanto, era esperado que un porcentaje siguiese esa línea de pensamiento, pero habría que realizarse un estudio más profundo para determinar los factores que inducen a ello.

Cuando comparamos los resultados de la percepción de acoso y la actitud respecto a mitos sobre agresión sexual podemos extraer que el promedio refería que el acoso callejero se había limitado de una a algunas veces en el pasado año y que estaba entre muy en desacuerdo a neutral respecto a los mitos sobre agresión sexual. Entonces, si bien la prevalencia de acoso concuerda con las cifras nacionales $(4,5,23)$ e internacionales $(6,8)$, demostrando ser un problema 
Percepción y actitudes frente al acoso sexual callejero en estudiantes mujeres de una Universidad Privada de Medicina

en la salud pública por sus repercusiones en el bienestar físico y mental de la población femenina joven, aún no se han tomado medidas para aplacarlo.

Si tomamos en cuenta el estudio de Durán (20) en el que explicaba que la razón del desbalance entre el número de agresiones sexuales y la cantidad de denuncias se debería a una desestimación por parte de la víctima influenciada por la ideología de su medio. Se podría inferir que en el caso del acoso callejero, por el hecho de ser aceptado como una "práctica cotidiana benigna" (21) se subestima por considerarlo como parte de nuestra idiosincrasia. Sin embargo, que la participante joven promedio no se halla satisfecho con las creencias que el medio inculca se puede considerar un paso hacia adelante.

\section{REFERENCIAS BIBLIOGRÁFICAS}

1. Integración. Mujer rompe tus barreras (14 de Diciembre 2014). [Online]. Available from: http://files.gruporpp.info/ integracion/249095443-mujer-rompe-tus-barreras-v2.pdf

2. Bardales, O.; Ortiz, Z. (2012). Hostigamiento sexual en mujeres y varones universitarios. Estudio exploratorio. MIMP, Lima.

3. Observatorio Contra el Acoso Callejero - OCAC (2015). $8^{\circ}$ Congreso Chileno de Sociología 2014 y Encuentro Pre-Alas 2015. Masculinidades y legitimaciones del acoso sexual callejero en Chile. Recurso web: http: / /www.ocacchile.org/ wp-content / uploads / 2015 / 04 / Masculinidades-ylegitimaci\%C3\%B3n-del-acoso-callejero-en-Chile.pdf

4. Vallejo r, Elizabeth. La violencia invisible: acoso sexual callejero en Lima metropolitana. [Online] Available from: http://www.ocacchile.org/wp-content/uploads / 2015/01/E. -Vallejo-Rivera-La-violencia-invisible-acososexual-callejero-en-Lima-metropolitana.pdf [Accessed 7 May 2015].

5. Instituto de opinión pública. Acoso Sexual Callejero .Estado de la opinión pública. [Online] 2013; Marzo 2013 (Año VIII). Available from: http://textos.pucp.edu.pe/pdf/2678.pdf [Accessed 6 May 2015].

6. Fileborn, Bianca. Conceptual understandings and prevalence of sexual harassment and street harassment. Australian Institute of Family Studies (2013). [Online]. Available from: http://aifs.gov.au/acssa/pubs/sheets/rs6/ [Accessed 1 May 2015]

7. Fairchild, K., Rudman, L. (2008). Everyday Stranger Harassment and Women's Objetification. Internet: Springer.

8. Sullivan, Harmony B. "Hey Lady, Youre Hot! Emotional and Cognitive Effects of Gender-Based Street Harassment on Women." Dissertation for a Doctor of Psychology at Indiana University of Pennsylvania, 2011.

9. Kearney, L. K. 2005. US Mexican American and Caucasian university students' experience of sexual harassment: The intertwining of power and culture. Dissertation Abstracts International: Section B: The Sciences and Engineering, 65(8-B), 4292.

10. Equal Opportunities Commission. The EOC Announces Results of "Sexual Harassment - Questionnaire Survey for Education
Sector 2014". [Online]. Available from:http:// www.eoc.org.hk/eoc/graphicsfolder/ShowContent.aspx?Ite $\mathrm{mID}=12793$ [Accessed 7 May 2015]

11. Herrera Enríquez, María del Carmen; Herrera, Antonio; Expósito, Francisca. ¡Stop Acoso! Reacciones de los hombres a la confrontación de las víctimas. The European Journal of Psychology Applied to Legal Context. 2014, 6(2): 45-52

12. Ho, I. K., Dinh, K. T., Bellefontaine, S. A., \& Irving, A. L. (2012). Sexual harassment and posttraumatic stress symptoms among Asian and White women. Journal of Aggression, Maltreatment \& Trauma, 21(1), 95-113.

13. Fileborn, Bianca. Conceptual understandings and prevalence of sexual harassment and street harassment. Australian Institute of Family Studies (2013). [Online]. Available from: http://aifs.gov.au/acssa/pubs/sheets/rs6/ [Accessed 1 May 2015]

14. Gerger, H., Kley, H., Bohner, G., \& Siebler, F. (2007). The Acceptance of Modern Myths about Sexual Aggression (AMMSA) scale: Development and validation in German and English. Aggressive Behavior, 33, 422-440.

15. Donovan, M.A., \& Drasgow, F.D. (1997, April). Establishing the measurement equivalence of a measure of sexual harassment across five samples: A multiple comparison of differential item functioning. Paper presented at the annual meeting of the Society for Industrial-Organizational Psychology, St. Louis, MO.

16. Romero-Sánchez, Mónica, López Megas, Jesús, CarreteroDios, Hugo, \& Rincón Neira, Liliana. Versión colombiana de la escala Acceptance of Modern Myths about Sexual Aggression: primeros análisis psicométricos. 2013. Revista Latinoamericana de Psicología, 45(1), 121-134.

17. Brownmiller, S. (1975). Against our will: Men, women and rape. New York: Simon and Schuster.

18. Burt, M.R. (1980). Cultural myths and supports of rape. Journal of Personality and Social Psychology, 38, 217-230.

19. Matos Marcelo, Sylvia. Programa de Lucha Integral contra la Violencia Familiar y Sexual en Ayacucho (2005). Línea de Base. Huamanga.

20. Durán Segura, María Mercedes, Megías, Jesús L., Moya, Miguel: Valoración social de la violencia sexual: el rol de los mitos sobre las agresiones sexuales. Comunicación en congreso. IX Congreso Iberoamericano de Ciencia, Tecnología y Género. 2012

21. Bourdieu, P.: La dominación masculina. Argentina: Anagrama. 2000.

Fuentes de financiamiento

Este artículo ha sido financiado por los autores.

Conflicto de interés

Los autores declaran no presentar ningún conflicto de interés.

Correspondencia:

Ruth Corazón Llerena Benites

Dirección: Mz W, Lt 17,Jr. Alicante. La Molina. Lima

Teléfono: 950880769

Correo electrónico: rcllb@hotmail.com

Recibido: 14 de diciembre de 2015 Aprobado: 12 de febrero de 2016 